\title{
Factors Affecting the Diagnostic Yield of Transbronchial Biopsy Using Endobronchial Ultrasonography with a Guide Sheath in Peripheral Lung Cancer
}

\author{
Shotaro Okachi ${ }^{1}$, Naoyuki Imai ${ }^{2}$, Kazuyoshi Imaizumi ${ }^{3}$, Shingo Iwano ${ }^{4}$, Masahiko Ando ${ }^{5}$, \\ Tetsunari Hase ${ }^{1}$, Hiromichi Aso ${ }^{1}$, Masahiro Morise ${ }^{1}$, Keiko Wakahara ${ }^{1}$, Satoru Ito ${ }^{1}$, \\ Naozumi Hashimoto ${ }^{1}$, Mitsuo Sato ${ }^{1}$, Masashi Kondo ${ }^{1}$ and Yoshinori Hasegawa ${ }^{1}$
}

\begin{abstract}
Objective Endobronchial ultrasonography with a guide sheath (EBUS-GS) and virtual bronchoscopic navigation (VBN) improves the diagnostic yield in patients with peripheral pulmonary lesions (PPLs). Most previous reports on EBUS-GS-guided transbronchial biopsy (TBB) have included patients with benign and malignant diseases. We aimed to determine the factors that predicted a successful diagnosis by EBUS-GS-guided TBB diagnostic in patients with small peripheral lung cancer, with a focus on the high-resolution computed tomography (HRCT) findings before bronchoscopy.

Methods We retrospectively reviewed the medical records of 173 consecutive patients with 175 small $(\leq 30$ $\mathrm{mm}$ ) PPLs who were diagnosed with primary lung cancer between June 2010 and October 2013 at Nagoya University Hospital. All patients underwent EBUS-GS-guided TBB with VBN using a ZioStation computer workstation (Ziosoft, Osaka, Japan). We analyzed the patient characteristics, HRCT findings, diagnostic yield, and the diagnostic factors in small peripheral lung carcinoma.

Results The EBUS probe position was within the PPL in 83 of the 175 lesions (47\%) and $112(64.0 \%)$ cases were successfully diagnosed by EBUS-GS-guided TBB. A univariate analysis revealed that the following factors were associated with a significantly higher diagnostic yield: CT bronchus sign positivity, a lesion of $>20 \mathrm{~mm}$ in diameter, a solid nodule, and a probe position that was within the lesion. The following factors were not significant: the lesion location, the number of biopsies, and the lung cancer histology. A multivariate analysis revealed that the following factors significantly affected the diagnostic yield: CT bronchus sign positivity [odds ratio $(\mathrm{OR})=2.479$ ]; a probe position that was within the lesion $(\mathrm{OR}=2.542)$; and a solid nodule $(\mathrm{OR}=2.304)$.

Conclusion The significant factors that were significantly associated with a successful diagnosis using EBUS-GS-guided TBB in small peripheral lung carcinoma were as follows: CT bronchus sign positivity, a solid nodule, and a probe position that was within the lesion.
\end{abstract}

Key words: EBUS-GS, lung cancer, virtual bronchoscopic navigation, diagnostic factor, CT bronchus sign

(Intern Med 55: 1705-1712, 2016)

(DOI: 10.2169/internalmedicine.55.6341)

\footnotetext{
${ }^{1}$ Department of Respiratory Medicine, Nagoya University Graduate School of Medicine, Japan, ${ }^{2}$ Respiratory Medicine, Gifu Prefectural Tajimi Hospital, Japan, ${ }^{3}$ Department of Respiratory Medicine, Fujita Health University, Japan, ${ }^{4}$ Department of Radiology, Nagoya University Graduate School of Medicine, Japan and ${ }^{5}$ Center for Advanced Medicine and Clinical Research, Nagoya University Hospital, Japan Received for publication August 10, 2015; Accepted for publication September 16, 2015

Correspondence to Dr. Shotaro Okachi, s.okachi@med.nagoya-u.ac.jp
} 


\section{Introduction}

Lung cancer is the leading cause of cancer death worldwide. Most lung cancer patients already have advanced disease at the time of their diagnosis. Advances in computed tomography (CT) equipment have resulted in the improved detection of peripheral pulmonary lesions (PPLs) and lung cancers (1). An early histological diagnosis is essential for the optimal treatment and management of lung carcinoma. Several diagnostic modalities, including conventional transbronchial biopsy (TBB) using fluoroscopy, CT-guided TBB, endobronchial ultrasonography with a guide sheath (EBUSGS)-guided TBB, virtual bronchoscopic navigation (VBN), transthoracic needle aspiration (TTNA), and surgical biopsy, can be applied to the diagnosis of PPL. Although the diagnostic yield of TTNA is high in the case of peripheral lung malignancies, it is associated with a higher incidence of pneumothorax, and occasionally causes air embolism and tumor seeding $(2,3)$. EBUS-GS and VBN have improved the PPL diagnostic yield $(4,5)$. The diagnostic yield of EBUSGS-guided TBB in small $(\leq 30 \mathrm{~mm})$ PPL has been reported to be $63-79 \%$ (4-8). The factors which affect the EBUS-GS diagnostic yield in PPL have been reported; however, some reports have indicated that the probe position is the only independent predictor of the EBUS-guided TBB diagnostic yield $(7,8)$. It is also controversial whether the findings from high-resolution computed tomography (HRCT) before bronchoscopy (e.g., lesion size, location, and CT bronchus sign) are significant diagnostic factors that can be used in addition to the EBUS image. Furthermore, most previous studies have included patients with various benign and malignant diseases, including tuberculosis, nontuberculous mycobacterial infection, organizing pneumonia, lymphoma, and metastatic lung tumors $(4,5,7,8)$. We therefore assessed the diagnostic yield of EBUS-GS-guided TBB and VBN using a computer workstation, ZioStation (Ziosoft, Osaka, Japan) in a study population that only included patients with small peripheral primary lung cancer, and examined the factors that affected EBUS visualization and diagnostic success, particularly in the evaluation of CT findings before bronchoscopy.

\section{Materials and Methods}

\section{Subjects}

Two hundred seventy-one small $(\leq 30 \mathrm{~mm})$ peripheral pulmonary lesions were examined in 269 patients who underwent EBUS-GS-guided TBB between June 2010 and October 2013 at Nagoya University Hospital. Ninety-six lesions which were diagnosed as lung metastases or benign diseases were excluded, and 175 lesions which were subsequently diagnosed as primary lung cancer in 173 patients were included (Fig. 1). At the time of the retrospective analysis, we recorded the characteristics of the nodules, including the size, location, the structure of the lesion, and the presence or absence of the CT bronchus sign (i.e., the finding of a bronchus leading directly to a PPL) (Fig. 2a and b) based on the HRCT findings (9). We divided PPLs into the following three groups according to their structures: solid nodule, pure ground-glass nodule (GGN), and part-solid nodule (Fig. 2c-e). PPLs were defined as lesions that were surrounded by the pulmonary parenchyma and which were not visible on bronchoscopy. The term "sub-solid nodule" was defined as a combination of pure GGN and part-solid nodule. Medical records were retrospectively reviewed in two groups: lesions that were diagnosed by EBUS-GS-guided TBB ( $\mathrm{n}=112$, diagnosed group) and undiagnosed lesions $(\mathrm{n}=$ 63, undiagnosed group). The definitive diagnoses of lung cancer were established in the undiagnosed lesions by endobronchial ultrasound-guided transbronchial needle aspiration (EBUS-TBNA), TTNA, video-assisted thoracoscopic surgery, or thoracotomy. Institutional review board approval was obtained (2014-0372). Individual patient informed consent was not required because of the retrospective nature of the study. Informed consent was obtained from each patient prior to bronchoscopy.

\section{CT examination and virtual bronchoscopic naviga- tion}

CT scans were performed at total lung capacity (inspiratory breath-hold) using 16-row or 64-row non-enhanced multi-detector CT (Aquilion; Toshiba Medical Systems, Tokyo, Japan). The HRCT data were reconstructed at $1 \mathrm{~mm}$ or $0.5 \mathrm{~mm}$ slice thickness with $0.5 \mathrm{~mm}$ or $0.4 \mathrm{~mm}$ intervals (16-row or 64-row, respectively) using a standard reconstruction algorithm. An individual CT dataset for each patient was sent to a ZioStation computer workstation. An experienced chest radiologist (S. Iwano) constructed all of the VBN images (from the carina tracheae to the leading bronchus of the objective lesion). The bronchoscopy operator could manipulate the VBN images (6). The pulmonary artery closest to the bronchus was used to predict the paths when the bronchus to the lesion was not visible on HRCT (10).

\section{The procedure and sedation}

Radial EBUS was performed using an endoscope ultrasonography system (EU-ME1; Olympus, Tokyo, Japan) equipped with $20 \mathrm{MHz}$ mechanical radial-type probes measuring $1.4 \mathrm{~mm}$ (UM-S20-17S; Olympus) or $1.7 \mathrm{~mm}$ (UMS20-20R; Olympus) in diameter. A thin bronchoscope (BFP260; Olympus, channel diameter: $2.0 \mathrm{~mm}$ ) and guide sheath (K-201; Olympus, external diameter: $1.95 \mathrm{~mm}$ ) were used for the 1.4-mm probe, and a thicker bronchoscope (BF1T260; Olympus, channel diameter: $2.8 \mathrm{~mm}$ ) and guide sheath (K-203; Olympus, external diameter: $2.55 \mathrm{~mm}$ ) were used for the $1.7-\mathrm{mm}$ probe. The probe and bronchoscope were selected by the operator.

The GS-covered EBUS probe was inserted through the working channel of the bronchoscope and advanced to the 


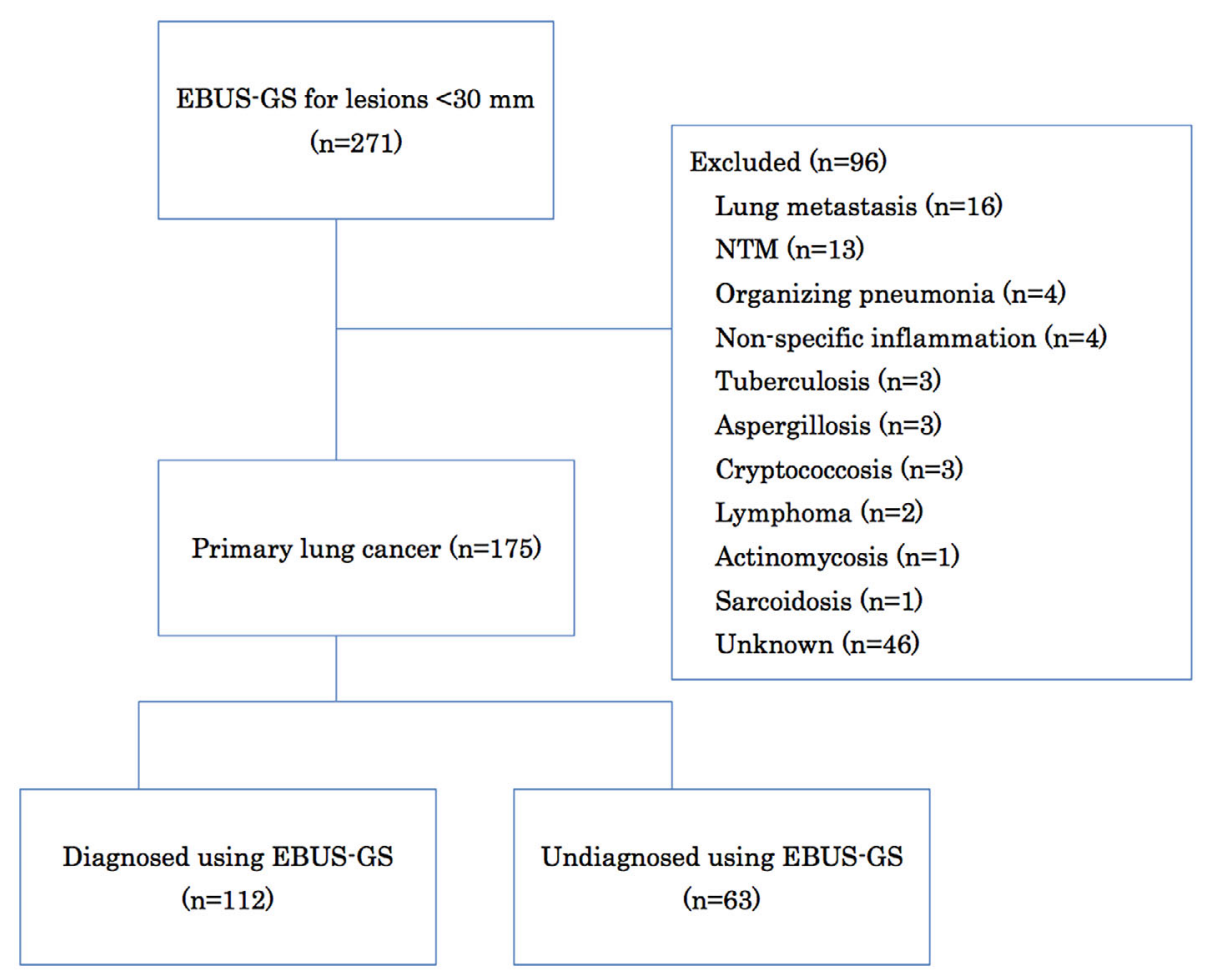

Figure 1. Flow diagram. Two hundred seventy-one small $(\leq 30 \mathrm{~mm})$ peripheral pulmonary lesions were examined by EBUS-GS-guided TBB. Ninety-six lesions, which were diagnosed as lung metastases or benign diseases, were excluded. The 175 lesions that were subsequently diagnosed as primary lung cancer were included in the present study.

PPL to obtain an EBUS image. The GS position was adjusted by radiographic fluoroscopy. If an EBUS image of PPL could not be visualized, the probe was removed from the GS and an angulated double-hinged curette (CC-6DR-1; Olympus) was used to reach the PPL. After the PPL was located on the EBUS image, the probe was removed to leave the guide sheath in the PPL. Biopsies were performed using forceps, with bronchial brushing, and washing through the GS under fluoroscopic guidance to obtain histological and cytological specimens; biopsies were repeated until an adequate number of specimens had been collected. As a rule, we obtained nine samples for each case. At all times during the procedure, the operators were able to refer to three monitors, which showed the actual bronchoscopic image, the VBN image, and the fluoroscopic image at the same time. All procedures were performed under local anesthesia and a safe method of sedation with individually calculated doses of intravenous midazolam $(11,12)$.

We classified the EBUS probe positions into the following three groups as described previously: [1] "within" (the probe was located within PPL); [2] "adjacent to" (the probe was located adjacent to PPL); and [3] "outside" (the probe was located outside PPL) (Fig. 2f-h) $(8,13,14)$.

In this study, we considered lesions with positive cytological findings or evident malignant findings on histological examination to have been positively diagnosed using EBUSGS-guided TBB.

\section{Statistical analysis}

Mann-Whitney $U$ tests (or $t$-tests if data were normally distributed) and Pearson's chi-square tests were used for the statistical analyses of continuous and categorical variables, respectively, to compare the diagnosed and undiagnosed lesions. Univariate and multivariate logistic regression analyses were performed to elucidate significant predictors of positive results on EBUS-GS-guided TBB or the "within" PPL EBUS probe position. The variables that were analyzed in relation to diagnosis using EBUS-GS-guided TBB were as follows: location ( $\mathrm{S} 1 / \mathrm{S} 2 / \mathrm{S} 1+2 / \mathrm{S} 6$ or the others), structure (solid nodule or sub-solid), size ( $>20 \mathrm{~mm}$ or $\leq 20 \mathrm{~mm}$ ), CT bronchus sign status (positive or negative), and EBUS image (within or adjacent to/outside). The variables that were analyzed in relation to the visualization of the EBUS image were as follows: location (S1/S2/S1+2/S6 or the others), structure (solid nodule or subsolid), size ( $>20 \mathrm{~mm}$ or $\leq 20$ $\mathrm{mm}$ ), and CT bronchus sign status (positive or negative). The diagnostic factors were compared using receiver operating characteristic (ROC) curves. $p$ values of $<0.05$ were considered to be statistically significant. All of the analyses were performed using the SPSS software program (version 22, IBM Corp., Armonk, USA).

\section{Results}

The characteristics of the lesions are shown in Table 1. Of 

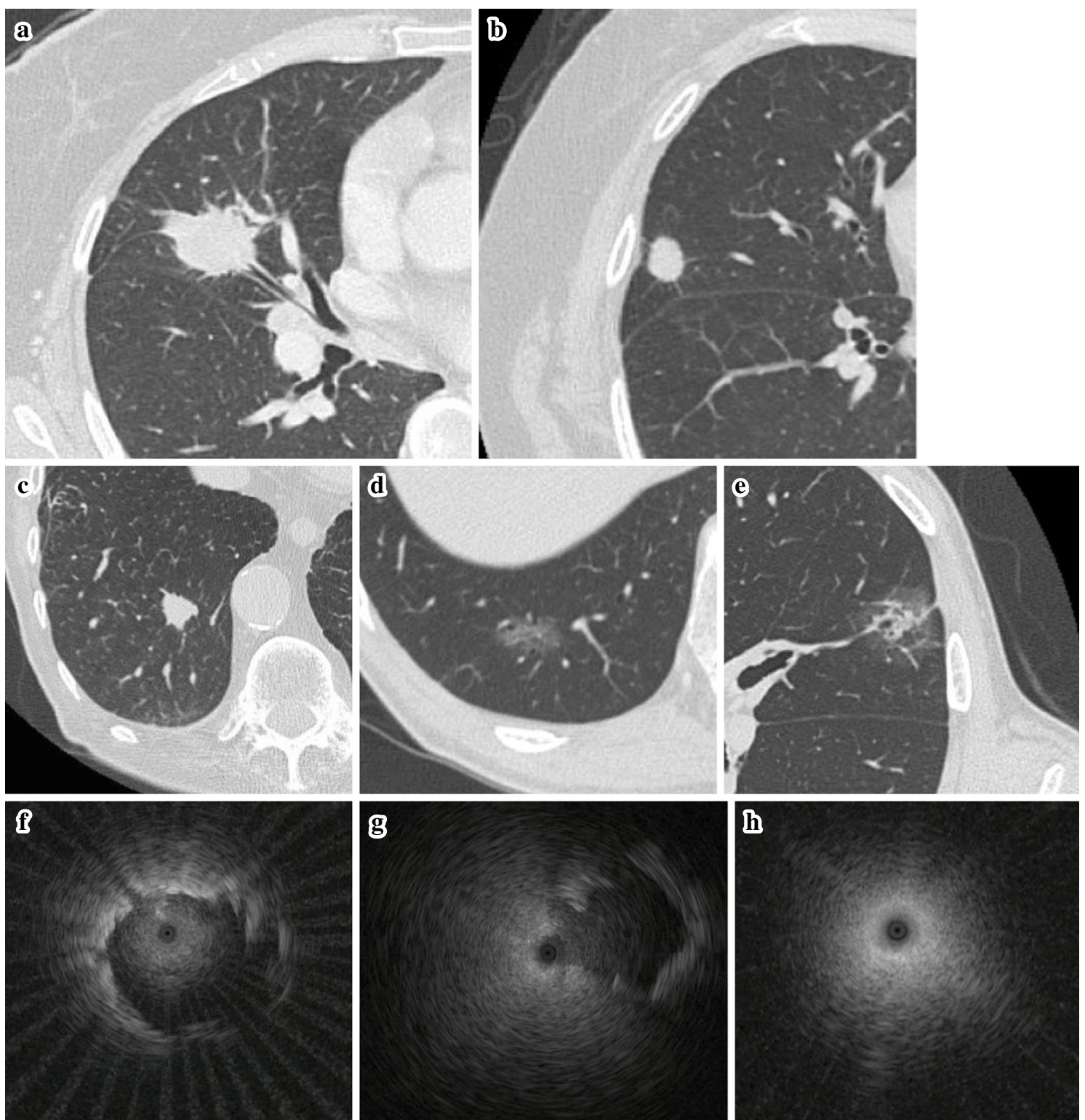

Figure 2. (a) A chest CT image revealing a bronchus leading directly to a PPL (CT bronchus signpositive). (b) A chest CT image revealing a bronchus that does not lead directly to a PPL (CT bronchus sign-negative). (c-e) We divided PPLs into the following three groups according to their structures: solid nodule (c), pure GGN (d), and part-solid nodule (e). (f) The EBUS probe is located within a PPL. (g) The EBUS probe is located adjacent to a PPL. (h) The EBUS probe is located outside a PPL.

the 175 lesions, 112 (64\%) were diagnosed using EBUSGS-guided TBB. The median age of the patients was 70 years, and 112 (64\%) were male. Ninety-three lesions (53\%) were located in the upper lobes. The CT appearances of the lesions were as follows: pure GGN $(n=7)$, part-solid nodules $(n=31)$, and solid nodules $(n=137)$. The median longest diameter of the lesions was $21 \mathrm{~mm}$ (range: 10-30), and 81 $(46 \%)$ of the lesions were $\leq 20 \mathrm{~mm}$. One hundred forty-four lesions (82\%) were CT bronchus sign-positive. Regarding the position of the probe, the probe was within the PPL in $83(47 \%)$ cases, adjacent to the PPL in $61(35 \%)$, and outside the PPL in $31(18 \%)$. One hundred sixty-four (94\%) procedures were performed using the $1.4-\mathrm{mm}$ probe. The median number of biopsy specimens was nine (range 2-14), and $120(69 \%)$ lesions were diagnosed as adenocarcinoma. There were no significant differences between the diagnosed and undiagnosed groups in terms of gender, location, EBUS probe, number of biopsies, or pathological diagnosis (Table 1).

A univariate analysis revealed that the structure of the lesions (solid nodule vs. sub-solid nodule), lesion size ( $>20$ $\mathrm{mm}$ vs. $\leq 20 \mathrm{~mm}$ ), CT bronchus sign (positive vs. negative), and EBUS image ("within" vs. "adjacent to" and "outside") were significant diagnostic factors. A multivariate analysis revealed that solid nodules [odds ratio $(\mathrm{OR})=2.304 ; 95 \%$ confidence interval $(\mathrm{CI})=1.069-4.968 ; \mathrm{p}=0.033$, CT bronchus sign positivity $(\mathrm{OR}=2.479 ; 95 \% \mathrm{CI}=1.072-5.734 ; \mathrm{p}=$ $0.034)$, and the "within" probe position $(\mathrm{OR}=2.542 ; 95 \%$ 
Table 1. Characteristics of the Lesions $(n=175)$.

\begin{tabular}{|c|c|c|c|c|c|}
\hline & & Total $(n=175)$ & Diagnosed $(n=112)$ & Undiagnosed $(n=63)$ & $\mathrm{p}$ valuc \\
\hline Age & Median (range) & $70(44-89)$ & $71(44-89)$ & $70(56-85)$ & 0.567 \\
\hline \multicolumn{6}{|l|}{ Gender } \\
\hline & Male & 112 & 71 & 41 & 0.79 \\
\hline & Female & 63 & 41 & 22 & \\
\hline \multicolumn{6}{|l|}{ Location } \\
\hline & Upper & 93 & 61 & 32 & 0.662 \\
\hline & Middle or lingular & 17 & 12 & 5 & \\
\hline & Lower & 65 & 39 & 26 & \\
\hline \multicolumn{6}{|l|}{ Structure } \\
\hline & Pure GGO & 7 & 3 & 4 & 0.061 \\
\hline & Part-solid nodule & 31 & 15 & 16 & \\
\hline & Solid nodule & 137 & 94 & 43 & \\
\hline \multirow[t]{3}{*}{ Size } & Median (range) & $21(10-30)$ & $22(10-30)$ & $19(10-30)$ & 0.012 \\
\hline & $\leq 20 \mathrm{~mm}$ & 81 & 45 & 36 & 0.031 \\
\hline & $21-30 \mathrm{~mm}$ & 94 & 67 & 27 & \\
\hline \multicolumn{6}{|l|}{$\mathrm{CT}$ bronchus sign } \\
\hline & Positive & 144 & 99 & 45 & 0.005 \\
\hline & Negative & 31 & 13 & 18 & \\
\hline \multicolumn{6}{|l|}{ EBUS image } \\
\hline & Within & 83 & 64 & 19 & $<0.001$ \\
\hline & Adjacent to & 61 & 42 & 19 & \\
\hline & Outside & 31 & 6 & 25 & \\
\hline \multicolumn{6}{|l|}{ EBUS probe } \\
\hline & 1.4-mm probe & 164 & 105 & 59 & 0.979 \\
\hline & 1.7-mm probe & 11 & 7 & 4 & \\
\hline Number of biopsy & Median (range) & $9(2-14)$ & $9(2-12)$ & $9(3-14)$ & 0.548 \\
\hline \multicolumn{6}{|l|}{ Diagnosis } \\
\hline & Adenocarcinoma & 120 & 78 & 42 & 0.36 \\
\hline & Squamous cell carcinoma & 24 & 16 & 8 & \\
\hline & Non-small cell carcinoma & 14 & 9 & 5 & \\
\hline & Small cell carcinoma & 14 & 9 & 5 & \\
\hline & Large cell carcinoma & 2 & 0 & 2 & \\
\hline & Adenosquamous cell carcinoma & 1 & 0 & 1 & \\
\hline
\end{tabular}

GGN: ground-glass nodule, CT: computed tomography, EBUS: endobronchial ultrasonography

Table 2. Logistic Regression Analysis of Factors Affecting Diagnostic Yield of EBUS-GS.

\begin{tabular}{|c|c|c|c|c|c|}
\hline \multirow[t]{2}{*}{ Variables } & & Univariate & & Multivariate & \\
\hline & & $\mathrm{OR}(95 \% \mathrm{CI})$ & $\mathrm{p}$ value & OR $(95 \% \mathrm{CI})$ & $\mathrm{p}$ value \\
\hline \multirow[t]{2}{*}{ Location } & $\mathrm{S} 1, \mathrm{~S} 2, \mathrm{~S} 1+2$ and $\mathrm{S} 6(\mathrm{n}=85)$ & $1.402(0.715-2.605)$ & 0.285 & - & - \\
\hline & The others $(n=90)$ & 1 (ref) & & & \\
\hline \multirow[t]{2}{*}{ Structure } & Solid nodule $(\mathrm{n}=137)$ & $2.429(1.168-5.050)$ & 0.017 & $2.304(1.069-4.968)$ & 0.033 \\
\hline & Subsolid nodule $(\mathrm{n}=38)$ & $1(\mathrm{ref})$ & & 1 (ref) & \\
\hline \multirow[t]{2}{*}{ Size } & $>20 \mathrm{~mm}(\mathrm{n}=94)$ & $1.985(1.062-3.712)$ & 0.032 & & \\
\hline & $\leq 20 \mathrm{~mm}(\mathrm{n}=81)$ & 1 (ref) & & & \\
\hline \multirow[t]{2}{*}{ CT bronchus sign } & Positive ( $n=144)$ & $3.046(1.375-6.750)$ & 0.006 & $2.479(1.072-5.734)$ & 0.034 \\
\hline & Negative $(n=31)$ & 1 (ref) & & 1 (ref) & \\
\hline \multirow[t]{2}{*}{ EBUS image } & Within $(n=83)$ & $3.088(1.603-5.947)$ & 0.001 & $2.542(1.285-5.026)$ & 0.007 \\
\hline & Adjacent to or outside $(n=92)$ & 1 (ref) & & 1 (ref) & \\
\hline
\end{tabular}

EBUS-GS: endobronchial ultrasonography with a guide sheath, OR: odds ratio, CI: confidence interval, ref: reference, CT: computed tomography

$\mathrm{CI}=1.285-5.026 ; \mathrm{p}=0.007)$ were significant predictors of a successful diagnosis using EBUS-GS-guided TBB (Table 2).

The probe position was within the PPL in 83 of the 175 (47\%) lesions. The univariate analysis showed that a positive CT bronchus sign (vs. negative) and lesion size $>20 \mathrm{~mm}$ (vs. $\leq 20 \mathrm{~mm}$ ) were significantly associated with an EBUS finding of "within" PPL. These two factors were also found to be statistically significant in the multivariate analysis. The lesion location and structure were not significantly associated with successful EBUS visualization (Table 3). 
Table 3. Logistic Regression Analysis of Factors for Visualization of EBUS.

\begin{tabular}{|c|c|c|c|c|c|c|c|}
\hline \multirow[t]{2}{*}{ Variables } & & \multirow[t]{2}{*}{ Within $(\mathrm{n}=83)$} & \multirow[t]{2}{*}{$\begin{array}{l}\text { Adjacent to or } \\
\text { outside }(n=92)\end{array}$} & \multicolumn{2}{|l|}{ Univariate } & \multicolumn{2}{|l|}{ Multivariate } \\
\hline & & & & OR $(95 \% \mathrm{CI})$ & $\mathrm{p}$ value & OR $(95 \% \mathrm{CI})$ & $\mathrm{p}$ value \\
\hline \multirow[t]{2}{*}{ Location } & $\mathrm{S} 1, \mathrm{~S} 2, \mathrm{~S} 1+2$ and $\mathrm{S} 6(\mathrm{n}=85)$ & 42 & 43 & $0.857(0.4737-1.552)$ & 0.61 & - & - \\
\hline & The others $(\mathrm{n}=90)$ & 41 & 49 & $1(\mathrm{ref})$ & & & \\
\hline \multirow[t]{2}{*}{ Structure } & Solid nodule $(n=137)$ & 69 & 68 & $1.739(0.830-3.643)$ & 0.142 & - & - \\
\hline & Subsolid nodule $(\mathrm{n}=38)$ & 14 & 24 & 1 (ref) & & & \\
\hline \multirow[t]{2}{*}{ Size } & $>20 \mathrm{~mm}(\mathrm{n}=94)$ & 26 & 55 & $3.259(1.747-6.079)$ & $<0.001$ & $3.120(1.649-5.905)$ & $<0.001$ \\
\hline & $\leq 20 \mathrm{~mm}(\mathrm{n}=81)$ & 57 & 37 & 1 (ref) & & 1 (ref) & \\
\hline \multirow[t]{2}{*}{ CT bronchus sign } & Positive $(n=144)$ & 76 & 68 & $3.832(1.553-9.456)$ & 0.004 & $3.568(1.407-9.048)$ & 0.007 \\
\hline & Negative $(\mathrm{n}=31)$ & 7 & 24 & $1(\mathrm{ref})$ & & 1 (ref) & \\
\hline
\end{tabular}

EBUS: endobronchial ultrasonography, OR: odds ratio, CI: confidence interval, ref: reference, CT: computed tomography

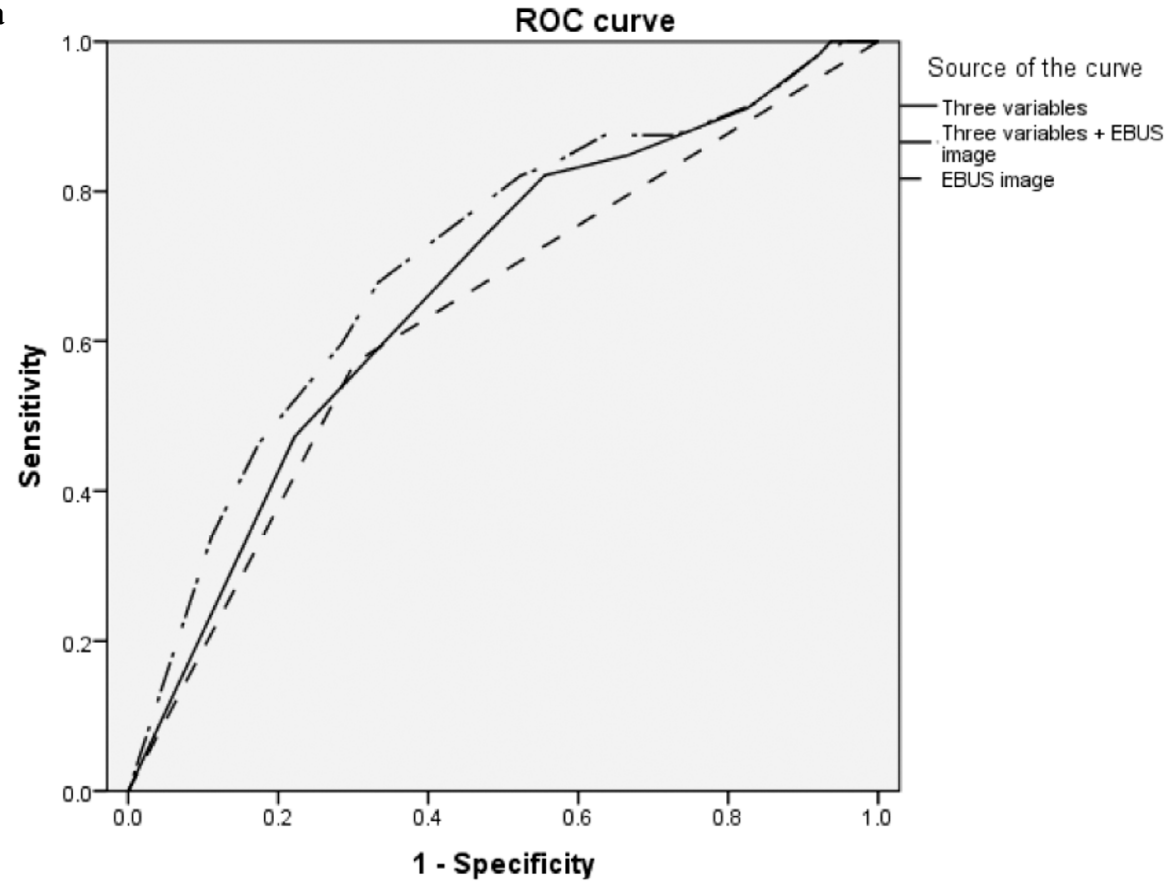

b

Area under ROC curve

\begin{tabular}{|l|c|}
\hline variables & Area \\
\hline Three variables & 0.670 \\
Three variables + EBUS image & 0.708 \\
EBUS image & 0.635 \\
\hline
\end{tabular}

Figure 3. (a) ROC curves comparing the EBUS image with a combination of three CT findings (structure, CT bronchus sign, and size) in relation to the EBUS-GS-guided TBB diagnostic yield. (b) The areas under the ROC curves for the EBUS image, the three variables, and the combination of the EBUS image and the three variables were $0.635,0.670$, and 0.708 , respectively.

Fig. 3 shows the ROC curves comparing the EBUS image with a combination of three CT findings (structure, CT bronchus sign status, and size) in relation to the EBUS-GSguided TBB diagnostic yield. The areas under the ROC curves for the EBUS image, the three variables, and that the combination of the EBUS image and three variables were
$0.635,0.670$, and 0.708 , respectively.

\section{Discussion}

Although there are previous reports which identify some of the factors that affect the EBUS-GS diagnostic yield, 
most previous studies have included patients with benign and malignant diseases or with lesions of $>30 \mathrm{~mm}$. Several previous reports have revealed a difference in the diagnostic yield in patients with benign and malignant diseases $(8,15)$. In the present study, we therefore restricted our patient selection to patients with small $(\leq 30 \mathrm{~mm})$ primary lung carcinoma. The results of our study revealed that the overall diagnostic yield was $64 \%$, and that a solid nodule structure, CT bronchus sign positivity, and the "within" PPL probe position were independently associated with a successful diagnosis. Furthermore, we showed that both the HRCT and EBUS findings were important for a successful diagnosis using the ROC curves.

Rivera et al. reported that conventional flexible bronchoscopy for peripheral lesions $\leq 20 \mathrm{~mm}$ and $>20 \mathrm{~mm}$ in diameter showed sensitivities of $34 \%$ and $63 \%$, respectively, whereas TBB using radial-EBUS showed a 56\% diagnostic yield in lesions of $\leq 20 \mathrm{~mm}$ and $78 \%$ in lesions of $>20 \mathrm{~mm}$ in a recently performed meta-analysis (2). The probe position was an important factor in predicting the diagnostic yield of EBUS-GS-guided TBB. Previous reports have indicated the probe position to be the only significant diagnostic factor $(7,8)$. In the multivariate analysis of the present study, the "within" PPL probe position was found to be significantly associated with a successful diagnosis using radial EBUS (OR=2.542; $\mathrm{p}=0.007$ ). With regard to EBUS imaging, Huang et al. reported that lesion size $(\leq 20 \mathrm{~mm}$ or $>20 \mathrm{~mm})$ is a significant factor affecting PPL visualization using EBUS; however, the CT bronchus sign was not included in their univariate or multivariate analyses (15). In the present study, a lesion size of $>20 \mathrm{~mm}$ and CT bronchus sign positivity were found to significantly associated with the successful visualization of the probe position within the lesion in the multivariate analysis $(p<0.001$ and $p=0.007$, respectively).

Our institution previously reported that the internal opacity of lesions is a significant diagnostic factor for PPL; however, the sensitivity of this factor was low for small nonsolid type lung carcinoma (6). Because GGNs are usually difficult to identify using radiographic fluoroscopy or EBUS, the TBB diagnostic yield may be low. Recently, some reports described the use of TBB with EBUS-GS, including different types of EBUS images, for the diagnosis of GGNs $(16,17)$. Izumo et al. investigated radial EBUS images, and showed a correlation between the blizzard sign (a subtle, but noticeable increase in intensity and a radius with a whitish acoustic shadow) and pure GGNs (16). The diagnostic yield of EBUS-guided TBB may be improved in GGNs with the use of new data.

Previous reports have revealed a higher conventional TBB diagnostic yield for patients with peripheral lung lesions in whom the CT bronchus sign is present $(9,18)$. CT bronchus sign positivity is significantly associated with visualization of the lesion using EBUS and a successful bronchoscopic diagnosis using EBUS-GS (19-21). In the present study CT bronchus sign positivity was found to be significantly asso- ciated with a successful diagnosis and with EBUS visualization in both the univariate and multivariate analyses.

Although multivariate analyses of two previously published studies indicated that lesion size is a statistically significant factor for the bronchoscopic diagnosis of PPL $(17,22)$, other studies have failed to corroborate these findings $(7,8)$. The univariate analysis of the present study showed that the diagnostic yield was significantly higher in lesions of $>20 \mathrm{~mm}$ in diameter in comparison to those of $\leq$ $20 \mathrm{~mm}$ in diameter; however, the multivariate analysis did not show any significance. Only $32 \%(25 / 79)$ of the lesions with diameters of $\leq 20 \mathrm{~mm}$ were visualized with the EBUS probe within the lesion. In contrast, $61 \%$ (57/94) of the lesions with diameters of $>20 \mathrm{~mm}$ were visualized with the EBUS probe $(\mathrm{p}<0.001)$. Although EBUS visualization is difficult in smaller PPLs, a successful diagnosis may be achieved, particularly in lesions that are visible using EBUS.

Shinagawa et al. reported that lesions located in the left S6 segment had significantly lower diagnostic sensitivity in comparison to lesions in other locations using an ultrathin bronchoscope under CT guidance (23). Yoshikawa et al. found that the diagnostic yields were significantly high for PPLs located in the right-middle lobe and the left lingular segment (22). Other studies indicate that it is difficult to reach lesions in the right-upper lung because the tips of EBUS catheters are relatively long and stiff, and are difficult to maneuver in tortuous airways $(14,24)$. We therefore divided the lesion locations into $\mathrm{S} 1, \mathrm{~S} 2, \mathrm{~S} 1+2$, and $\mathrm{S} 6$ or others, based on the assumption that it is not easy to reach lesions in the former segments; however, we did not find any significant differences in the diagnostic yield among the different locations.

Although the EBUS image is a highly significant factor for a successful diagnosis using EBUS-GS, we showed that HRCT findings before bronchoscopy are important. The area under the curve for the combined EBUS image and three factors regarding the HRCT findings were superior to those for the EBUS image or the three factors alone (area under the curve 0.708 vs. 0.635 and 0.670 , respectively; Fig. 3). Thus, CT and EBUS findings are important for the successful diagnosis of small peripheral lung cancer using EBUSGS-guided biopsy. The lung cancer diagnostic yield was 84.4\% when pre-procedure HRCT revealed a solid and larger nodule with CT bronchus sign positivity and the EBUS image during bronchoscopy showed a "within" PPL probe position. This is a considerably high diagnostic yield; however, there were some unsuccessful cases. It is therefore essential to consider additional diagnostic modalities based on multidisciplinary discussions when anticipated results are not obtained.

Virtual bronchoscopy (VB) is a CT-based imaging technique which allows a noninvasive intraluminal evaluation of the tracheobronchial tree and which is used as a guide to navigate the bronchoscope near a target lesion $(4,6,10)$. Ishida et al. reported that VBN improved the diagnostic yield while decreasing the overall duration of the examina- 
tion (25). In a recent study, Asano et al. revealed that VBN improved the diagnostic yield for lesions showing involved bronchi on CT images (21). In the present study, all patients underwent bronchoscopy with VBN and CT bronchus sign positivity was independently associated with a successful diagnosis.

Our study is associated with some limitations. Our conclusions may not be generalized because the study was retrospective in nature and because it was performed at a single medical institution. Our study included only seven pure GGN lesions and no lesions of $<10 \mathrm{~mm}$ in diameter. Smaller lesions $(<15 \mathrm{~mm})$ and pure GGN cases have a lower diagnostic yield $(6,8,17)$. It is therefore possible that lesions that were fit for TBB-based diagnosis were selected.

\section{Conclusion}

In conclusion, the CT findings, including CT bronchus sign positivity and lesion structure and EBUS visualization for EBUS-GS-guided biopsy were significant diagnostic factors in patients with small peripheral lung cancer. Therefore, chest physicians must carefully evaluate patients' HRCT findings on an individual basis before performing bronchoscopy to assess the best way to diagnose a PPL.

\section{The authors state that they have no Conflict of Interest (COI).}

\section{Acknowledgement}

We are indebted to all of the participating clinicians. The present study was supported in part by a Grant-in-Aid for scientific research from the Japan Society for the Promotion of Science (Grant Number 15K19282).

\section{References}

1. Bach PB, Mirkin JN, Oliver TK, et al. Benefits and harms of CT screening for lung cancer: a systematic review. JAMA 307: 24182429, 2012.

2. Rivera MP, Mehta AC, Wahidi MM. Establishing the diagnosis of lung cancer: Diagnosis and management of lung cancer, 3rd ed: American college of chest physicians evidence-based clinical practice guidelines. Chest 143 (5 Suppl): e142S-e165S, 2013.

3. Tomiyama N, Yasuhara Y, Nakajima Y, et al. CT-guided needle biopsy of lung lesions: A survey of severe complication based on 9783 biopsies in Japan. Eur J Radiol 59: 60-64, 2006.

4. Asahina H, Yamazaki K, Onodera Y, et al. Transbronchial biopsy using endobronchial ultrasonography with a guide sheath and virtual bronchoscopic navigation. Chest 128: 1761-1765, 2005.

5. Asano F, Matsuno Y, Tsuzuku A, et al. Diagnosis of peripheral pulmonary lesions using a bronchoscope insertion guidance system combined with endobronchial ultrasonography with a guide sheath. Lung Cancer 60: 366-373, 2008.

6. Iwano S, Imaizumi K, Okada T, Hasegawa Y, Naganawa S. Virtual bronchoscopy-guided transbronchial biopsy for aiding the diagnosis of peripheral lung cancer. Eur J Radiol 79: 155-159, 2011.

7. Tamiya M, Okamoto N, Sasada S, et al. Diagnostic yield of combined bronchoscopy and endobronchial ultrasonography, under LungPoint guidance for small peripheral pulmonary lesions. Respirology 18: 834-839, 2013.
8. Yamada N, Yamazaki K, Kurimoto N, et al. Factors related to diagnostic yield of transbronchial biopsy using endobronchial ultrasonography with a guide sheath in small peripheral pulmonary lesions. Chest 132: 603-608, 2007.

9. Naidich DP, Sussman R, Kutcher WL, Aranda CP, Garay SM, Ettenger NA. Solitary pulmonary nodules. CT-bronchoscopic correlation. Chest 93: 595-598, 1988.

10. Onodera $Y$, Omatsu T, Takeuchi $S$, et al. Enhanced virtual bronchoscopy using the pulmonary artery: improvement in route mapping for ultraselective transbronchial lung biopsy. AJR Am J Roentgenol 183: 1103-1110, 2004.

11. Ogawa T, Imaizumi K, Hashimoto I. Prospective analysis of efficacy and safety of an individualized-midazolam-dosing protocol for sedation during prolonged bronchoscopy. Respir Investig 52: 153-159, 2013.

12. Okachi $\mathrm{S}$, Imai $\mathrm{N}$, Imaizumi $\mathrm{K}$, et al. Endobronchial ultrasound transbronchial needle aspiration in older people. Geriatr Gerontol Int 13: 986-992, 2013.

13. Kurimoto N, Miyazawa T, Okimasa $\mathrm{S}$, et al. Endobronchial ultrasonography using a guide sheath increases the ability to diagnose peripheral pulmonary lesions endoscopically. Chest 126: 959-965, 2004.

14. Shirakawa T, Imamura F, Hamamoto J, et al. Usefulness of endobronchial ultrasonography for transbronchial lung biopsies of peripheral lung lesions. Respiration 71: 260-268, 2004.

15. Huang CT, Ho CC, Tsai YJ, Yu CJ, Yang PC. Factors influencing visibility and diagnostic yield of transbronchial biopsy using endobronchial ultrasound in peripheral pulmonary lesions. Respirology 14: 859-864, 2009.

16. Izumo T, Sasada S, Chavez C, Matsumoto Y, Tsuchida T. Radial endobronchial ultrasound images for ground-glass opacity pulmonary lesions. Eur Respir J 45: 1-8, 2015.

17. Ikezawa $Y$, Sukoh N, Shinagawa N, Nakano K, Oizumi S, Nishimura M. Endobronchial ultrasonography with a guide sheath for pure or mixed ground-glass opacity lesions. Respiration $\mathbf{8 8}$ : 137-143, 2014.

18. Gaeta M, Pandolfo I, Volta S, et al. Bronchus sign on CT in peripheral carcinoma of the lung: value in predicting results of transbronchial biopsy. AJR Am J Roentgenol 157: 1181-1185, 1991.

19. Minezawa $T$, Okamura $T$, Yatsuya $H$, et al. Bronchus sign on thinsection computed tomography is a powerful predictive factor for successful transbronchial biopsy using endobronchial ultrasound with a guide sheath for small peripheral lung lesions: a retrospective observational study. BMC Med Imaging 15: 21, 2015.

20. Evison M, Crosbie PA, Morris J, Martin J, Barber PV, Booton R. Can computed tomography characteristics predict outcomes in patients undergoing radial endobronchial ultrasound-guided biopsy of peripheral lung lesions? J Thorac Oncol 9: 1393-1397, 2014.

21. Asano F, Shinagawa N, Ishida $T$, et al. Virtual bronchoscopic navigation improves the diagnostic yield of radial-endobronchial ultrasound for peripheral pulmonary lesions with involved bronchi on CT. Intern Med 54: 1021-1025, 2015.

22. Yoshikawa M, Sukoh N, Yamazaki K, et al. Diagnostic value of endobronchial ultrasonography with a guide sheath for peripheral pulmonary lesions without X-ray fluoroscopy. Chest 131: 17881793, 2007.

23. Shinagawa N, Yamazaki K, Onodera Y, et al. Factors related to diagnostic sensitivity using an ultrathin bronchoscope under CT guidance. Chest 131: 549-553, 2007.

24. Herth FJ, Ernst A, Becker HD. Endobronchial ultrasound-guided transbronchial lung biopsy in solitary pulmonary nodules and peripheral lesions. Eur Respir J 20: 972-974, 2002.

25. Ishida T, Asano F, Yamazaki K, et al. Virtual bronchoscopic navigation combined with endobronchial ultrasound to diagnose small peripheral pulmonary lesions: a randomised trial. Thorax 66 : 1072-1077, 2011.

(C) 2016 The Japanese Society of Internal Medicine http://www.naika.or.jp/imonline/index.html 